P. C. H. Martens, S. Tsuruta, and M. A. Weber, eds.

\title{
Hard X-ray Spectrum of the Above-the-Looptop Source in Impulsive Solar Flares
}

\author{
S. Masuda \\ Solar-Terrestrial Environment Laboratory, Nagoya University, \\ Toyokawa, Aichi 442-8507, Japan
}

\section{Extended Abstract}

The Hard X-ray Telescope (HXT: Kosugi et al. 1991) onboard Yohkoh has observed that, in impulsive solar flares, a hard X-ray source is located above the apex of a soft X-ray flaring loop, in addition to double footpoint sources (Masuda et al. 1994, 1995). This observation suggests that flare energy-release, probably magnetic reconnection, takes place not in the soft X-ray loop but above the loop. It is important to derive the hard X-ray spectrum of the above-the-looptop source accurately in order to understand how electrons are energized there. The above-the-looptop source was most clearly observed during the 13 January 1992 flare. However, the count rate, especially in the $\mathrm{H}$-band $(53-93 \mathrm{keV})$, is too small to synthesize high-quality images and to derive an accurate spectrum.

An X2.8-class impulsive flare (hereafter F1) occurred slightly behind the east limb, around 8:20 UT on 18 August 1998. The most outstanding characteristic is that an above-the-looptop source is clearly observed in the H-band for the first time (Figure 1). This provides us with accurate spectral information for this source by using all of the energy bands (14-23-33-53-93 keV). It is found that the HXT count rates are well explained by emission from an isothermal plasma whose temperature and emission measure are $\sim 200 \mathrm{MK}$ and $\sim 1 \times 10^{45} \mathrm{~cm}^{-3}$, respectively.

We also analyzed an X4.9 flare (F2) which occurred about 14 hours later in the same active region. In F2, the above-the-looptop source is not observed in any energy band (Figure 1). This may not indicate a difference in the energy release mechanism between these two flares, but a difference of the observational situation. Actually, the hard X-ray intensity of the footpoint sources in F2 is about one order of magnitude greater than that of $F 1$ though the intensities of the so-called looptop gradual source and the soft X-ray loop are almost similar in the two flares. This may mean that most of each of the footpoint sources in F1 is occulted by the limb. These observations suggest that the intensity ratio between above-the-looptop sources and footpoint sources in the H-band is usually much greater than the HXT dynamic range, $\sim 10$. This might be the main reason that the above-the-looptop source had never been observed in the $\mathrm{H}$-band before F1 was observed. To investigate the above-the-looptop source in detail, we need intense flares which occur at the appropriate location, i.e., slightly behind the limb. 


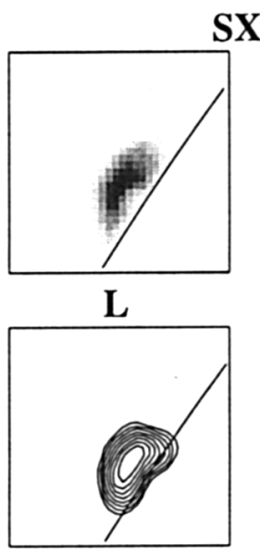

M2

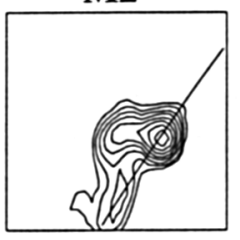

SXR

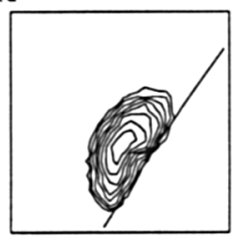

M1

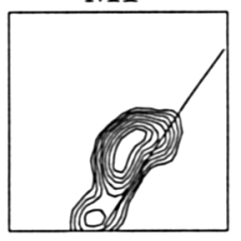

H

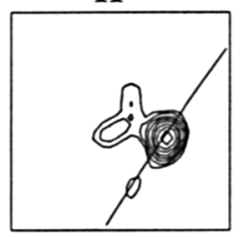

SXR

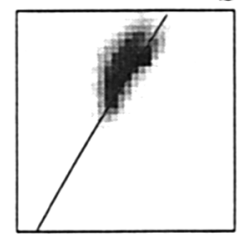

L

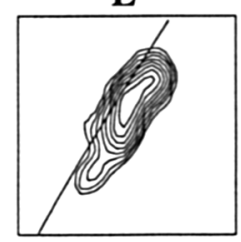

M2

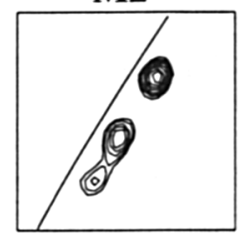

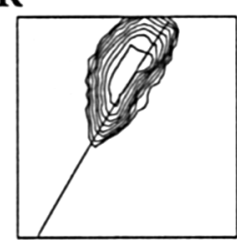

M1

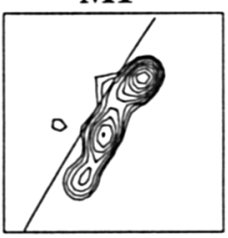

H

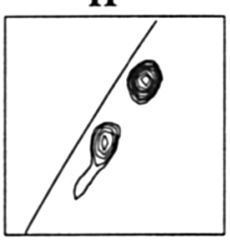

Figure 1. Left: Soft and hard X-ray images of F1. The upper two panels (grey scale and contours) show a soft X-ray image from Yohkoh/SXT (Be-filter) at 8:21:37 UT. The bottom four panels are hard X-ray images in the four HXT energy bands (14-23-33-53$93 \mathrm{keV})$. The photon accumulation time is 8:19:33-8:19:57 UT, and the FOV is $78 \times 78$ arcsec. The solid line indicates the solar limb. The contour levels are $6.25,8.84,12.5,17.7,25.0,35.4,50.0$, and $70.7 \%$ of the maximum counts. Right: The same as the left figure, but for F2. The soft X-ray image is taken at 22:17:11 UT. The photon accumulation time is $22: 15: 33-22: 15: 34 \mathrm{UT}$.

\section{References}

Kosugi, T., et al. 1991, Solar Phys., 136, 17

Masuda, S., Kosugi, T., Hara, H., Sakao, T., Shibata, K., \& Tsuneta, S. 1995, PASJ, 47, 677

Masuda, S., Kosugi, T., Hara, H., Tsuneta, S., \& Ogawara, Y. 1994, Nature, 371,495 